\title{
Temperature Effect on Hydroxyapatite Preparation by Co-precipitation Method under Carbamide Influence
}

\author{
Jing Luo ${ }^{1, a}$, Juan Chen, Wenzhao Li, Zhiliang Huang ${ }^{b}$, Changlian Chen \\ ${ }^{1}$ Department of Material and Engineering Science, Wuhan Institute of Technology, Wuhan 430074, Hubei, China
}

\begin{abstract}
Hydroxyapatite crystal was prepared by homogeneous co-precipitation method using a mixture of Calcium nitrate and diammonium phosphate as raw materials and carbamide as the buffering agent. To analyzed the influence of temperature on hydroxyapatite crystal morphology, the phase composition, crystal morphology and growth orientation were characterized by X-ray diffraction, scanning electron microscope and infrared spectrum respectively. The results show that when the reaction temperature increase from $70{ }^{\circ} \mathrm{C}$ to $95{ }^{\circ} \mathrm{C}$, the phases composition of crystal transform from the two phases of tricalcium phosphate and eight calcium phosphate to hydroxyapatite single crystal; the morphology of the hydroxyapatite crystals transforms from nodular whisker to perfectly and compactly acicular whisker.
\end{abstract}

\section{Introduction}

Hydroxyapatite(abbr. as HAP or HA) is a kind of calcium phosphate with weak alkaline, slightly soluble in water; the chemical formula is Ca10(PO4)6(OH)2. Its composition and structure are basically the same with biological Apatite; it belongs to the hexagonal system. With its excellent bioactivity and biocompatibility, it can combine closely with bone formation, guide the growth of bone, it is a kind of biomedical materials to obtain excellent comprehensive properties[1-3].At present, the preparation methods of hydroxyapatite are as the followings: hydrolysis, hydrothermal synthesis[4], ultrasonic irradiation[5], precipitation[6],solgel[7-8] and micro-emulsion[9].

Precipitation method becomes one of the preferred methods for the preparation of hydroxyapatite because of its advantages of the simple process, low cost, and the crystallization degree and particle size of powders are controllable[10]. The homogeneous co-precipitation method which is used in this article, joining precipitant on the basis of precipitation method, the precipitating agent dissolves in solution evenly and the reactive ion generates slowly with the chemical reaction happens. By this way, it can overcome the local inhomogeneity of precipitant which is caused by directly joining precipitating agent in the solution from the outside. The study found that the reaction temperature has certain influence on the grain structure and crystal morphology[11].

According to other reports we know that the influencing factors in the preparation of hydroxyapatite include reaction Temperature, $\mathrm{pH}$, reaction promoter , molar ratio of calcium phosphate and reaction temperature. And the aspect research about the influence of reaction temperature reported less, therefore, the author researches on the influence of the reaction temperature in preparation of hydroxyapatite by homogeneous co-precipitation method.

\section{Experimental}

\subsection{Experiment reagent and instrument}

The chemical medicines which are chosen in this experiment are shown in table 1 below.

\subsection{Experiment method}

In this experiment, using $\mathrm{Ca}(\mathrm{NO} 3) 2 \cdot 4 \mathrm{H} 2 \mathrm{O}$ as the calcium source, (NH4)2HPO4 as a source of phosphorus, carbamide as precipitant and tetrahydrofuran as reaction promoter(which is used to lead the growth of acicular whisker), preparing hydroxyapatite powder by homogeneous co-precipitation method. [12]:

The basic principles for the response are shown below

$\mathrm{CO}(\mathrm{NH} 2) 2+\mathrm{H} 2 \mathrm{O}==\mathrm{CO} 2+2 \mathrm{NH} 3$

$\mathrm{Ca}(\mathrm{NO} 3) 2 \cdot 4 \mathrm{H} 2 \mathrm{O}+(\mathrm{NH} 4) 2 \mathrm{HPO} 4+\mathrm{NH} 3 \cdot \mathrm{H} 2 \mathrm{O} \rightarrow$ $\mathrm{Ca} 10(\mathrm{PO} 4) 6(\mathrm{OH}) 2 \downarrow$

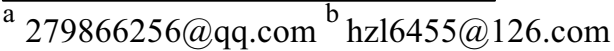


Table 1. The chemicals used in preparing hydroxyapatite powder by homogeneous co-precipitation method

\begin{tabular}{|c|c|c|c|}
\hline experiment reagent & molecular formula & molecular weight & purity \\
\hline Calcium Nitrate Tetrahydrate & $\mathrm{Ca}\left(\mathrm{NO}_{3}\right)_{2} \cdot 4 \mathrm{H}_{2} \mathrm{O}$ & 236.15 & $99 \%$ \\
\hline diammonium phosphate & $\left(\mathrm{NH}_{4}\right)_{2} \mathrm{HPO}_{4}$ & 132.06 & $98.5 \%$ \\
\hline carbamide & $\mathrm{CO}\left(\mathrm{NH}_{2}\right)_{2}$ & 60.06 & $99 \%$ \\
\hline concentrated nitric acid & $\mathrm{HNO}_{3}$ & 63 & $65 \% \sim 68 \%$ \\
\hline tetrahydrofuran & $\mathrm{C}_{4} \mathrm{H}_{8} \mathrm{O}$ & 72.11 & $99 \%$ \\
\hline anhydrous alcohol & $\mathrm{C}_{2} \mathrm{H}_{5} \mathrm{OH}$ & 46.07 & $99.7 \%$ \\
\hline distilled water & $\mathrm{H}_{2} \mathrm{O}$ & 18.00 & \\
\hline
\end{tabular}

Specific reaction steps are as follows: first of all, weigh quantitative (NH4)2HPO4 and $\mathrm{Ca}(\mathrm{NO} 3) 2 \cdot 4 \mathrm{H} 2 \mathrm{O}$ dissolved in distilled water respectively as the starting solutions, making sure the molar ratio of $\mathrm{Ca} 2+$ : $\mathrm{PO} 43-$ was $1.67: 1$. Next, adding the (NH4)2HPO4 solution into the $\mathrm{Ca}(\mathrm{NO} 3) 2 \cdot 4 \mathrm{H} 2 \mathrm{O}$ solution at a constant speed slowly. Stir constantly, when the solution is mixed uniformly, adding dilute nitric acid (HNO3)(offering $\mathrm{H}+$ to dissolve $\mathrm{Ca} 3(\mathrm{PO} 4) 2$ precipitation) solution which is prepared beforehand into the solution and stirring till the precipitation completely dissolved.

After that, adding a moderate amount of carbamide (additive that includes amine) as buffer. Adjusting the $\mathrm{pH}$ of the reaction solution with dilute nitric acid (HNO3) solution, making the initial $\mathrm{pH}$ of solution is 3 and adding a moderate amount of tetrahydrofuran into it.

Then pouring the solution into a $100 \mathrm{ml}$ volumetric flask, diluting with distilled water to volume, and mixing. Taking $25 \mathrm{ml}$ solution into the reaction reactor heats under different temperature $\left(\mathrm{A}\left(70{ }^{\circ} \mathrm{C}\right), \mathrm{B}\left(75^{\circ} \mathrm{C}\right), \mathrm{C}\right.$ $\left.\left(80{ }^{\circ} \mathrm{C}\right), \mathrm{D}\left(85{ }^{\circ} \mathrm{C}\right), \mathrm{E}\left(90{ }^{\circ} \mathrm{C}\right), \mathrm{F}\left(95{ }^{\circ} \mathrm{C}\right)\right)$ for 24 hours. Taking out the reaction reactor after heating, making it cool naturally. After cooling, standing it for 12 hours at room temperature.

Removing the reactant, filtering it and washing it with distilled water for many times and anhydrous alcohol for three times.Next taking the filter residue into the vacuum drying oven, drying at $60{ }^{\circ} \mathrm{C}$ for 24 hours, obtaining the solid samples.

Finally, calcining the solid sample at $900{ }^{\circ} \mathrm{C}$ for 2 hours, getting the samples which are numbered $\mathrm{a} \sim \mathrm{f}$.

\section{Result and discussion}

Figure 1 is the XRD patterns of powder samples which were prepared under different conditions, respectively. It can clearly be seen from the patterns that when reaction temperature rise from $70{ }^{\circ} \mathrm{C}$ to $95{ }^{\circ} \mathrm{C}$, the phase of the powder transforms from OCPA to OCPA and OCP, then to HAP. With the rise of reaction temperature, OCPA and OCP phases are obvious reduce and disappeared. Meanwhile, the crystal crystallinity of HAP phase increases significantly. The diffraction peak is sharp and clear, the peak shape, peak height are up to standard, shows that the HAP crystal tends to be purely. Comparing the XRD of sample $f$ with the standard card (JCPDS No. 09-0432), it suggests that it is the pure samples of HAP. Moreover, there is no impurity peak in the XRD images, proving that the phase is single HAP
[13].We can conclude that the increase of reaction temperature is helpful to obtain high purity of HAP crystal.

Figure 2 is the SEM photographs of powder samples which were prepared under different conditions, respectively. It can clearly be seen from the images that when reaction temperature rise from $70{ }^{\circ} \mathrm{C}$ to $95{ }^{\circ} \mathrm{C}$, the crystal grows from nodular whisker to compactly acicular whisker. It can also be seen from the images that the whisker growth in the form of the bundle. Monolithic whisker is acicular with a smooth surface. This is because of the use of tetrahydrofuran. The Hydrogen bonding ability of different organic reaction promoter on HAP lead it to different morphology. The influence of surface energy in different surfaces can determine the relative development degree of the crystal. By this way, we can get the acicular morphology of HAP crystal we need. The length between 30 to $60 \mu \mathrm{m}$, about 40 microns, the average length of about 2 to 4 microns wide, length to diameter ratio between 10 to 20 .

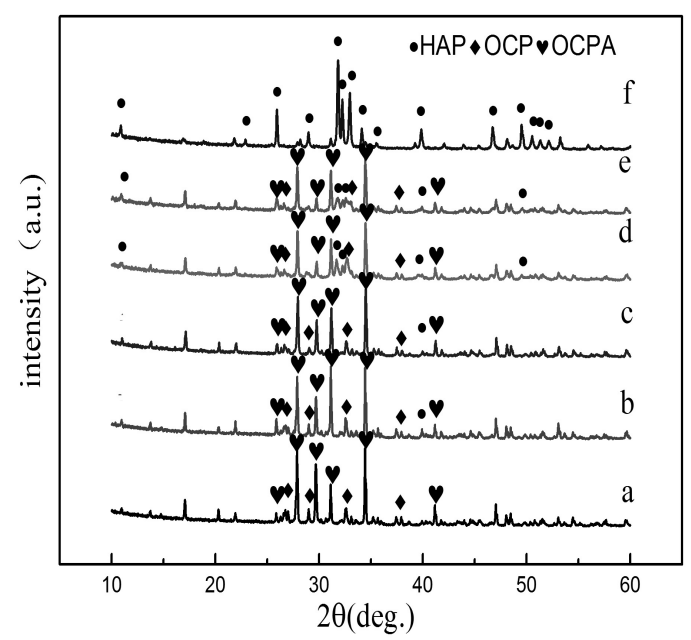

Figure. 1. The XRD spectra of the HAP samples in different temperature

a: sample a; b: sample b; c: sample c;

d: sample d; e: sample e; f: sample f 


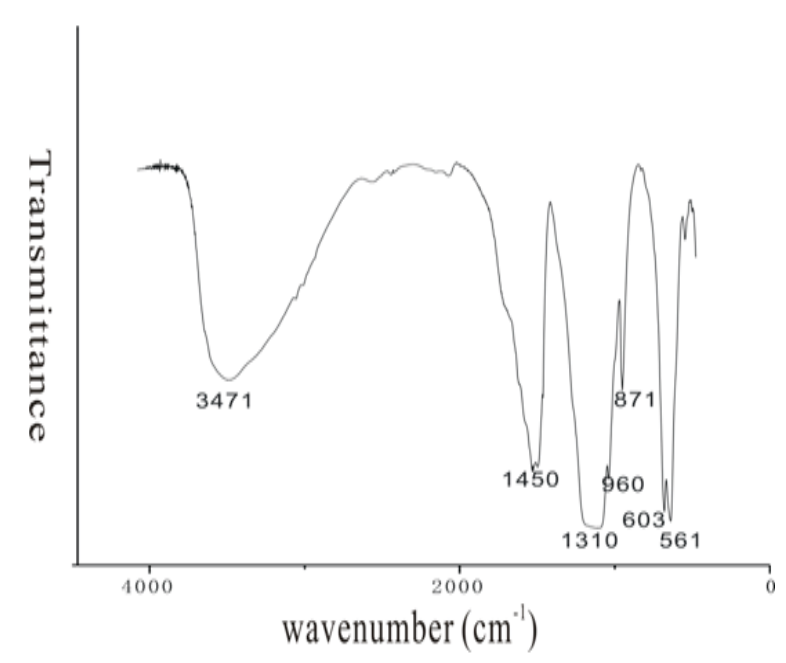

Figure. 2. FT-IR spectrum of sample $f$

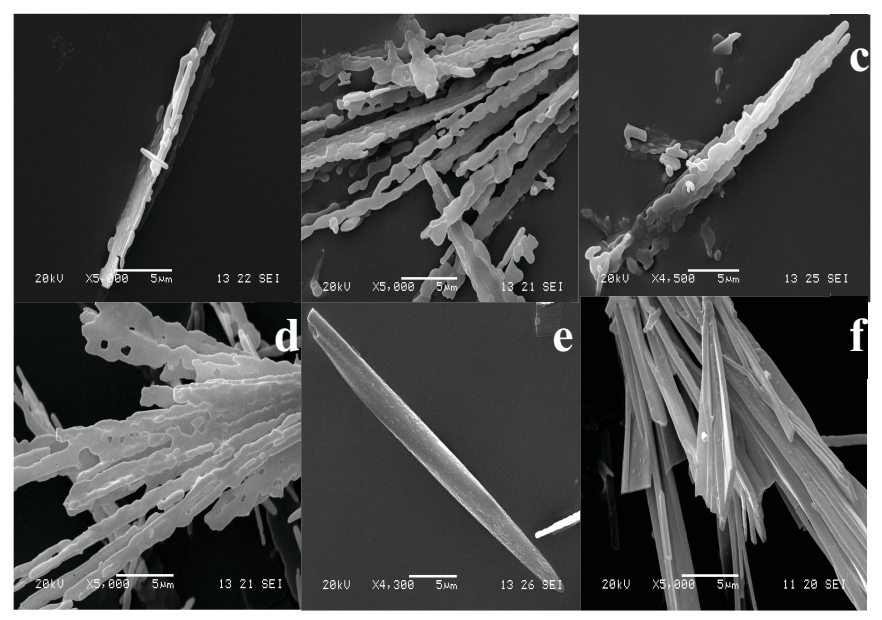

Figure. 3. The SEM photographs of the HAP samples at different pressures

a: sample a; b: sample b; c: sample c;

d: sample d; e: sample e; f: sample f

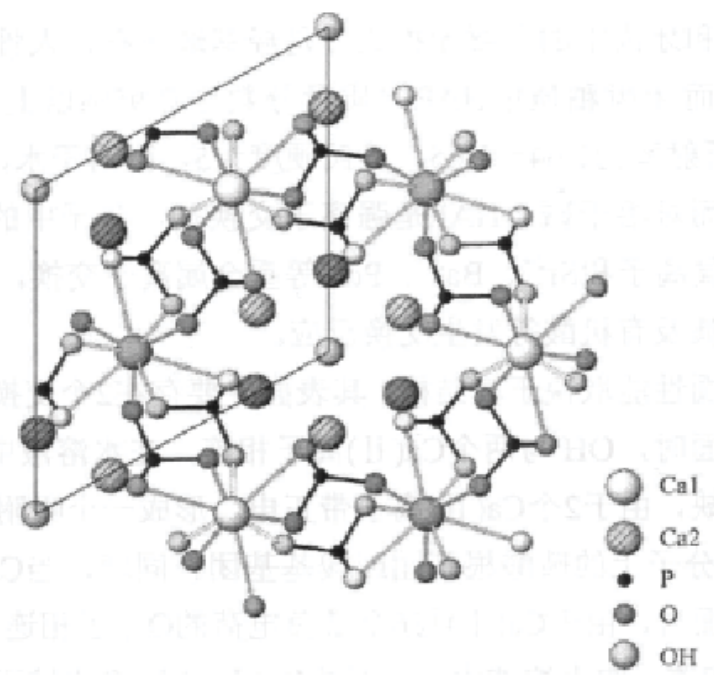

Figure. 4. Projection of hydroxyapatite crystal structure on (0001) plane [15]

Figure 3 is the infrared spectrum of sample $f$. Seen from the image, there are several characteristic peaks. The absorption peaks at $561 \mathrm{~cm}-1,960 \mathrm{~cm}-1,603 \mathrm{~cm}-$
1 are the PO43 - characteristics vibration peak; at $871 \mathrm{~cm}$ - 1 is the HPO42- characteristics vibration peak; at 1450 $\mathrm{cm}$ - 1 belongs to the characteristics infrared spectrum(type B replace) of $\mathrm{CO} 32-[14]$; OH - is shown by the characteristic of vibration peak at $1310 \mathrm{~cm} \mathrm{-1}$ without $3570 \mathrm{~cm}-1$. It can prove that the [PO4] tetrahedron is replaced by the $[\mathrm{CO} 3 \cdot \mathrm{OH}]$ coordinative tetrahedron .And the absorption peaks at $3421 \mathrm{~cm}-1$ belongs to characteristics infrared spectrum of $\mathrm{H} 2 \mathrm{O}$. The analysis above show that part of the $\mathrm{OH}$ - was replaced by the CO32- in the preparation of hydroxyapatite by homogeneous co-precipitation method. And it may because the use of carbamide.

Figure 4 is the crystal structure of hydroxyapatite. Combining it with the experimental results, we can make the following inferences. In the process of the formation of hydroxyapatite, firstly, generating $\mathrm{PO} \_4_{\text {tetrahedron, }}$ form the tetrahedral vacancies and octahedral vacancies; secondly, calcium ions occupy some vacancies to form tricalcium phosphate, and then forming eight calcium phosphate when vacancies are filled up; finally the hydroxyls enter into the structure and generating hydroxyapatite. In the final step, the temperature is an important factor. At the temperature of $95{ }^{\circ} \mathrm{C}$, the $\mathrm{OH}$ can be released smoothly by carbamide so that the HAP crystal can form completely.

\section{Conclusions}

The results show that with the rise of reaction temperature, the crystal we obtain becomes complete and pure. In order to get HAP crystal of high purity and complete morphology, the reaction temperature should be $95{ }^{\circ} \mathrm{C}$.

\section{Acknowledgments}

This project are supported by the National Natural Science Foundation of China (Grant No.51374155), the National Key Technology R\&D Program (Grant No. 2013BAB07B01), the State Key Program of National Natural Science of Hubei province (Grant No. 2014BCB034), and the National Natural Science Foundation of Hubei province of China (Grant No. 2014CFB796).

\section{References}

1. Deisinger U, Stenzel F, Ziegler G. Hydroxyapatite ceramics with tailored pore structure [J]. Key Engineering Materials, (2004), 264-268: 2047-2050.

2. Fleisch H, Russell $R$ G, Francis $M$ D. Diphosphonates inhibit hydroxyapatite dissolution in vitro and bone resorption in tissue culture and in vivo [J]. Science, (1969), 165: 1262-1264.

3. Kokubo T, Kim H M, Kawashita M. Novel bioactive materials with different mechanical properties $[\mathrm{J}]$. Biomaterials, (2003), 24(13): 2161-2175.

4. Sadat-Shojai M, Atai M, Nodehi A, et al. Hydroxyapatite nanorods as novel fillers for 
improving the properties of dental adhesives Synthesis and application [J].Dent Master,(2010),26(5):471-482.

5. HanYingchao, LiShipu, WangXinyu. A novel thermolysis method of colloidal protein precursors to prepare hydroxyapatite nanocrystals [J]. Cryst Res Technol, (2009),44(3):336-340.

6. Kothapalli C, Wei M, Vasilieve A, et al. Influence of temeperature and concentration on the sintering behavior and mechanical properties of hydroxyapatite [J].Acta Materialia, (2004), 52(19): 5655-5663.

7. LinRunrong, MaoXuan, YuQicong, et al. Preparation of bioactive nano-hydroxyapatite coating for artificial cornea [J].Current Aplied Physics,(2007),7(1):85-89.

8. Fathi M H, Hanifi A, Mortazavi V. Preparation and bioactivity evaluation of bone-like hydroxyapatite nanopowder [J].Journal of Materials Processing Technology,(2008),202(1/2/3):536-542.

9. Joachim Koetz, Kornelia Gawilitza, Sabine Kosmella. Formation of organically and inorganically passivated $\mathrm{CdS}$ nanoparticles in reverse microemulsions [J].Colloid Polym Sci,(2010),288(3):257-263.
10. Dorozhkin S V. Nanodimensional and nanocrystalline apatites and other calcium orthophosphates in biomedical engineering, biology and medicine [J]. Materials, (2009), 2(4):1975-2045

11. MinNaiBen. The physical basis of crystal growth (M). Shanghai science and technology press, 1982:339-372

12. Kong L B, Ma J, Boey F. Nanosized hydroxyapatite powders derived from coprecipitation process [J].Journal of Materials Science,2002,37(6):11311134

13. K.Kandori, N. Horigami, A. Yasukawa et al, Texture and Formation Mechanism of Fibrous Calcium Hydroxyapatite Particles Prepared by Decomposition of Calcium-EDTA Chelates [J]. J Am. Ceram. Soc., 1997, 80(5):1157-1164.

14. Zhiliang Huang, Dawei Wang, Yu Liu et al. FT-IR Investigation on Crystal Chemistry of Various CO32--Substituted Hydroxyapatite Solid Solutions $[\mathrm{J}]$. Chinese ournJal of Inorganic Chemistry, 2002, (5): 469-474.

15. Ager III J W, Balooch G., Ritchie R O. Fracture, aging, and disease in bone [J]. J Mater Res, 2006, 21 (8): 1878-1892 